DOI: https://doi.org/10.11144/Javeriana.umed58-3.apro

\title{
Aproximaciones a un estimativo de la capacidad para formar el talento humano en el campo de la salud en un centro médico académico
}

\section{Approaches to an Estimation of the Capacity to Form Human Talent in the Field of Health in an Academic Health Center}

Fecha de recepción: 06 Agosto 2017 | Fecha de aprobación: 06 Diciembre 2017 a Autor de correspondencia. Correo electrónico: crbrando@husi.org.co

Cómo citar: Brando CR, Castellanos Ramírez JC. Aproximaciones a un estimativo de la capacidad para formar el talento humano en el campo de la salud en un centro médico académico. Univ Med. 2017;58(3):1-9. doi: https://doi.org/10.11144/Javer iana.umed58-3.apro

\section{RESUMEN}

Uno de los mayores retos en la formación del talento humano en salud son los cálculos relacionados con la capacidad instalada de los centros de práctica para lograr la adecuada exposición al ambiente clínico y facilitar el logro de competencias. El artículo presenta un análisis desde la visión de un centro médico académico como es el Hospital Universitario San Ignacio en relación con la Pontificia Universidad Javeriana de Colombia. Palabras clave

capacidad instalada; talento humano en salud; centro médico académico.

\section{ABSTRACT}

One of the greatest challenges in the formation of human talent in health is the calculations related to the installed capacity of the practice centers to achieve adequate exposure to the clinical environment and facilitate the achievement of competencies. We present an analysis from the vision of an Academic Health Center such as the San Ignacio University Hospital in relation to the Pontificia Universidad Javeriana, Colombia.

Keywords

installed capacity; human talent in health; academic health center.

\section{Introducción}

Los centros o escenarios de práctica clínica son un componente esencial de la formación del talento humano en el área de la salud. Con miras a desarrollar programas de alta calidad, en el mundo se ha planteado el reto de establecer modelos que respondan a las necesidades cambiantes de los diversos sistemas y brinden suficientes espacios con características administrativas y clínicas que permitan el ejercicio simultáneo y sinérgico de la asistencia, la docencia, la investigación y la extensión. En los países con sistemas 
de educación en salud más avanzados, esta función recae en los centros médicos académicos (CMA). Por definición, son instituciones prestadoras de servicios de salud que comparten con la universidad la misión axiológica de formar a los profesionales de la salud. Además, en estos sitios se desarrollan múltiples actividades universitarias relacionadas con el campo de la salud. Entre ellos, se encuentran los CMA de las universidades de Duke, en Estados Unidos, y de Maastricht, en Holanda [1], así como el CMA de la Universidad Nacional de Singapur [2].

La separación de educación y atención sanitaria, habitual en algunos entornos, conduce a que el proceso de aprobación de nuevos programas o facultades priorice los aspectos educativo y académico, y relegue a un segundo plano el proceso de formación en el ambiente clínico. El concepto de CMA subsana esta división, puesto que brinda una formación trasversal que conjuga la educación en ciencias básicas, a cargo de la facultad y de la universidad, con un acercamiento progresivo a los pacientes, que comienza desde los primeros semestres de las carreras. Esta aproximación parte de experiencias de contacto y reconocimiento del entorno y culmina con las rotaciones clínicas. En Colombia se dificulta la aplicación de este modelo porque, pese a que en el país existen importantes instituciones educativas y de salud públicas, una gran parte de las responsabilidades y del volumen de los servicios de los sectores público y privado recaen en las instituciones privadas.

Este artículo propone una cuantificación aproximada de los recursos clínicos requeridos para la adecuada formación de los profesionales sanitarios, teniendo en cuenta la proporción necesaria de estudiantes respecto a los escenarios en que se lleva a cabo la práctica. El artículo no pretende generalizar, sino aprovechar la trayectoria educativa de la Pontificia Universidad Javeriana en el campo de la salud, para construir un modelo trazable, viable, sostenible y estandarizado. El artículo aspira a que esta sea la propuesta inicial para una generar una discusión encaminada a crear dicho modelo.

\section{Antecedentes}

El Hospital Universitario San Ignacio es un CMA de Bogotá que tiene una estrecha relación con la Pontificia Universidad Javeriana, no solo porque es la entidad que lo fundó, sino porque comparte con ella la misión de formar el talento humano en el área de la salud. El componente ambulatorio del hospital atiende necesidades asistenciales de nivel primario. Se trata de la Fundación Javeriana de Servicios Médicos Odontológicos Interuniversitarios Carlos Márquez Villegas (Javesalud), fundada hace veinte años, también por la Pontificia Universidad Javeriana.

La Pontificia es una de las instituciones de educación superior más antiguas y reconocidas de Colombia. Es también una de las 114 universidades que dirige la Compañía de Jesús en el mundo. La universidad cuenta con 18 facultades, 14 institutos y 62 departamentos que ofrecen 39 carreras y 125 programas de posgrado. Tiene más de 18.000 alumnos en pregrado y 4000 en posgrado. Su cuerpo docente está conformado por 3300 profesores, y su plantilla administrativa, por 1700 empleados.

La Facultad de Medicina cuenta con un programa de pregrado, 19 programas de especialización, 12 subespecializaciones, una maestría y un programa de Doctorado en Ciencias Biológicas, que concede gran importancia a las áreas de la genética y de las enfermedades infecciosas.

La Facultad de Medicina ha formado profesionales durante más de setenta años. Los egresados de sus programas de pre- y posgrado son reconocidos por su liderazgo, idoneidad y profesionalismo. La mayoría son destacados especialistas en las diferentes áreas del conocimiento médico, tanto en Colombia como en el exterior. No en vano, el Ranking QS de 2015 la clasificó como la primera facultad de medicina de Colombia [3].

En Colombia, la relación entre centros de práctica e instituciones educativas es "atípica" respecto a los estándares internacionales, que se definen mediante convenios reglamentados por normas que permiten cubrir las necesidades mediante el cumplimiento de requisitos, sin 
referentes de capacidad y calidad. La cuestión se puede abordar buscando indicadores de referencia que permitan determinar, entre otros aspectos, el número de alumnos por cama, el número de consultas y el número de alumnos por docente, entendiendo que todos ellos son los factores determinantes para definir los entornos adecuados para el aprendizaje. Estos enfoques no son nuevos y la literatura hace referencia a ellos. En España, para el curso 2000-2001, ingresaron 4471 estudiantes de primer año de medicina. Los centros de salud disponibles tenían 67.331 camas de hospital en 112 hospitales y 234 centros de atención primaria. Hubo un promedio de 4,49 camas de enseñanza por alumno. Hubo 3081 profesores titulares y asociados. Casi la mitad enseñaba en el ciclo preclínico y cerca de la mitad, en el ciclo clínico. También hubo 4492 profesores asistentes clínicos. Hoy, la relación media alumno-profesor en las facultades de medicina españolas es de 3,02 por 1,0 , con variaciones de entre 6,22 y 1,04 [4].

En otros países, la planeación va más allá. Desde la concepción misma del CMA, se tiene en cuenta el tamaño del hospital en el que se formará a los futuros profesionales. Veamos dos ejemplos:

Nepal [5]: se establece una relación de 6 camas por cada 100 alumnos admitidos anualmente, esto es, una ocupación de, al menos, el $70 \%$. El número de consultas ambulatorias debe ser, al menos, de 600 al día. En ese mismo orden de ideas, se expone la distribución por servicios de este hipotético CMA (tabla 1).
Tabla 1.Distribución por servicios del centro médico académico planeado

\begin{tabular}{|c|c|c|}
\hline Departamentos & $\begin{array}{l}\text { Núm. total de } \\
\text { camas }=600\end{array}$ & Servicios especializados \\
\hline Medicina interna & 120 & $\begin{array}{l}\text { Unidad coronaria. Servicios de endoscopia } \\
\text { digestiva. Gastroenterologia. Cardiología. }\end{array}$ \\
\hline Cirugía general & 120 & $\begin{array}{l}\text { Quemados. Servicio de urología. Cirugía } \\
\text { laparoscópica. }\end{array}$ \\
\hline $\begin{array}{l}\text { Obstetricia y } \\
\text { ginecologia }\end{array}$ & 80 & $\begin{array}{l}\text { Servicios de oncología ginecológicos. } \\
\text { Servicios de salud reproductiva. }\end{array}$ \\
\hline Ortopedia & 80 & $\begin{array}{l}\text { Ortopedia. Trauma. Servicios de lesiones } \\
\text { espinales. }\end{array}$ \\
\hline Pediatría & 80 & $\begin{array}{l}\text { Unidad de cuidados intensivos. Servicios de } \\
\text { inmunización. Pediatría y unidad de cuidado } \\
\text { intensivo neonatal. }\end{array}$ \\
\hline Oftalmología & 20 & FACO. \\
\hline Otorrinolaringología & 20 & Audiometría. \\
\hline Psiquiatría & 20 & Rehabilitación por abuso de drogas. \\
\hline Dermatología & 20 & \\
\hline Cirugia dental & 5 & \\
\hline Urgencias/observación & 25 & Manejo de emergencias de víctimas en masa. \\
\hline Anestesiología & 5 & \\
\hline
\end{tabular}

Además, para cada uno de los servicios propuestos, la proyección determina el número de docentes y su grado de reconocimiento académico (tabla 2).

Tabla 2.Propuesta de docentes por servicio según lo planeado

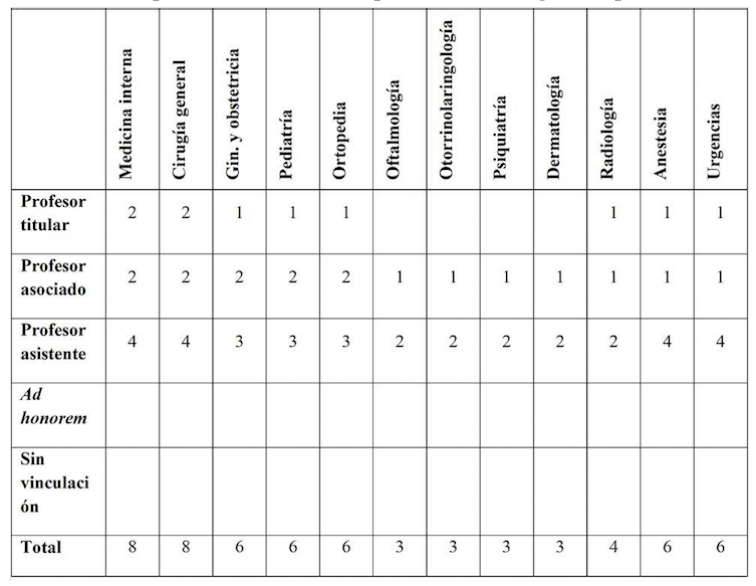

India: se plantea una situación similar a la de Nepal. El documento Minimum Standard Requirements for the Medical College for 150 Admissions Annually Regulations, 1999, del Medical Council of India [6], considera todos los aspectos e, incluso, prevé el tamaño de los servicios, las oficinas y los auditorios. El documento indica que la cantidad de consultas ambulatorias debe ser de, mínimo, 8 pacientes diarios (viejos y nuevos) por alumno y que 
la ocupación media anual de las camas será, mínimo, del $80 \%$.

En Latinoamérica no existe un consenso sobre la concentración de alumnos en los campos de práctica. En Perú se habla de 5 alumnos por cama hospitalaria [7], lo que permite concluir que habrá sobredemanda.

En Chile se establecen principios muy claros a través del proceso de asignación de cupos para alumnos de pregrado, normalizado por resoluciones estatales [8]. Las determinaciones al respecto se basan en un análisis muy completo de la producción asistencial del campo de práctica. La tabla 3 muestra la asignación numérica [9].

Tabla 3.Asignación numérica de cupos para alumnos

\begin{tabular}{|l|l|}
\hline Atención cerrada & 2 estudiantes por cama clínica \\
\hline Atención abierta & 2 estudiantes por unidad de atención \\
\hline $\begin{array}{l}\text { Servicios de imagenología, } \\
\text { Medicina física y rehabilitación }\end{array}$ & 1 estudiante por Box de atención o procedimiento \\
\hline Atención primaria & 3 estudiantes por unidad de atención \\
\hline
\end{tabular}

Nota* Se entiende por unidad de atención la dependencia física donde un profesional de la salud presta atención directa a un paciente ambulatorio.

La norma mexicana [10] hace hincapié en las condiciones del centro de prácticas y establece las siguientes precisiones numéricas:

En las áreas de hospitalización, las actividades de enseñanza clínica deben realizarse con un máximo de cinco alumnos por paciente y por profesor.

En consultorios, las actividades de enseñanza deben realizarse con un máximo de tres alumnos.

La Norma Técnica Ecuatoriana [11] establece el número máximo de alumnos, según se ve en la tabla 4.
Tabla 4.Máximo de cupos por alumnos según la norma ecuatoriana

\begin{tabular}{|l|l|}
\hline \multicolumn{1}{|c|}{ Tipo de atención } & \multicolumn{1}{|c|}{ Número máximo de alumnos } \\
\hline Hospitalización & $\begin{array}{l}\text { Un estudiante de pregrado por cama ocupada, en función de la } \\
\text { tasa de ocupación de la UAD, en jornadas de, máximo, 4 horas. } \\
\text { Dos médicos residentes y un interno rotativo por cada tratante a } \\
\text { cargo de un paciente. } \\
\text { Cada establecimiento debe aplicar del modo más conveniente } \\
\text { estas determinaciones numéricas, respetando el principio de } \\
\text { calidad en la atención y teniendo en cuenta que no deben saturarse } \\
\text { las habitaciones de los pacientes. }\end{array}$ \\
\hline En consulta externa & $\begin{array}{l}\text { Dos estudiantes de pregrado por cada consultorio, por cada } \\
\text { jornada de 4 horas. }\end{array}$ \\
\hline En quirófanos & $\begin{array}{l}\text { Cuatro estudiantes máximo, 2 de ellos de posgrado (uno de } \\
\text { anestesia y uno de cirugia), un interno rotativo y un estudiante de } \\
\text { enfermería en cada cirugia; podrían ser parte del equipo } \\
\text { quirúrgico. }\end{array}$ \\
\hline $\begin{array}{l}\text { En sala de partos y } \\
\text { centro obstétrico }\end{array}$ & $\begin{array}{l}\text { Un médico residente, un interno rotativo, un estudiante (medicina } \\
\text { u obstetricia) por cada 3 camas de labor; un estudiante para } \\
\text { atención de parto; un estudiante para cada 5 camas de } \\
\text { recuperación posparto. }\end{array}$ \\
\hline $\begin{array}{l}\text { En la sala de } \\
\text { emergencias }\end{array}$ & $\begin{array}{l}\text { Un estudiante por cubiculo de atención o procedimiento; un } \\
\text { residente y un interno rotativo por cada 4 cubículos de atención. }\end{array}$ \\
\hline $\begin{array}{l}\text { En los servicios de } \\
\text { imagenologia, } \\
\text { medicina fisica y } \\
\text { rehabilitación o } \\
\text { quinesiología }\end{array}$ & $\begin{array}{l}\text { Un estudiante por cada 2 cubículos de atención o procedimiento. } \\
\text { Este estudiante puede ser de pregrado o postgrado. }\end{array}$ \\
\hline
\end{tabular}

Al hacer una proyección de su capacidad instalada para 2009, la institución prestadora de servicios de salud (IPS) universitaria de la Universidad de Antioquia [12] propone que dicha capacidad se ajuste a las siguientes proporciones de alumnos por camas hospitalarias:

Estudiantes de pregrado de medicina: un estudiante por tres camas. Internos de medicina: un interno por 10 camas (para cada especialidad). Residente de medicina: un residente por 20 camas (para cada especialidad).

Estudiantes enfermería (práctica clínica): un estudiante por 4 camas. Estudiantes de enfermería (práctica administrativa): un estudiante por 15 camas.

Auxiliares de enfermería (práctica clínica): 2 estudiantes por 10 camas. Auxiliares de enfermería (práctica administrativa): un estudiante por 10 camas.

Para servicios de urgencias:

Medicina: estudiante de medicina de pregrado: un estudiante por cada 2 camas. Internos de medicina: 3 internos por cada turno de 12 horas. Residente de medicina: un residente por especialidad, por cada turno de 12 horas.

Enfermería: estudiante de enfermería (práctica administrativa): un estudiante por 10 cubículos. 
Enfermería (práctica clínica): un estudiante por 4 camas.

Auxiliar de enfermería (pra\#ctica cli\# nica): 2 estudiantes por 10 camas. Auxiliares de enfermería (práctica administrativa): un estudiante por 10 camas.

En Norteamérica, tenemos los ejemplos del CMA de la Universidad de Duke [13], en Durham, y el de la Universidad de Arkansas [14]. Las dos universidades divulgan el trabajo de sus CMA, reconocidos como dos de los diez mejores centros de formación de los Estados Unidos. En la tabla 5 presentamos la experiencia de estos dos centros.

Tabla 5.Hechos y datos de los centros médicos de las universidades de Duke y de Arkansas

\begin{tabular}{|l|r|r|}
\hline \multicolumn{1}{|c|}{ Datos } & Duke University & $\begin{array}{l}\text { University of } \\
\text { Arkansas }\end{array}$ \\
\hline Profesores clínicos de medicina & 1925 & 1429 \\
\hline Profesores de ciencias básicas & 211 & \\
\hline Profesores de escuela de enfermería & 90 & \\
\hline Estudiantes de medicina & 430 & 694 \\
\hline Residentes y fellows & 1006 & 789 \\
\hline Otros estudiantes de ciencias de la salud & 578 & \\
\hline \multicolumn{1}{|c|}{ Centro médico académico } & $\begin{array}{r}\text { Duke University } \\
\text { Medical Center }\end{array}$ & UAMC \\
\hline Camas & 957 & 450 \\
\hline Porcentaje ocupacional & $\ldots$ & \\
\hline Empleados & 7638 & \\
\hline Promedio de pacientes hospitalizados & 750 & \\
\hline Egresos & 40.326 & 26.216 \\
\hline Consulta externa & 1.063 .837 & 434.707 \\
\hline Consultas de urgencias & 70.701 & 58.179 \\
\hline Cirugías & 40.056 & 16.261 \\
\hline Número de laboratorios clínicos & 5.660 .804 & \\
\hline Partos & 3127 & \\
\hline
\end{tabular}

En el caso de España, tomamos la información del 2013 (tabla 6), publicada por el Observatorio de Resultados de Hospitales del Servicio Madrileño de Salud [15].

Tabla 6.Indicadores de hospitales de Madrid (España), 2011-2013

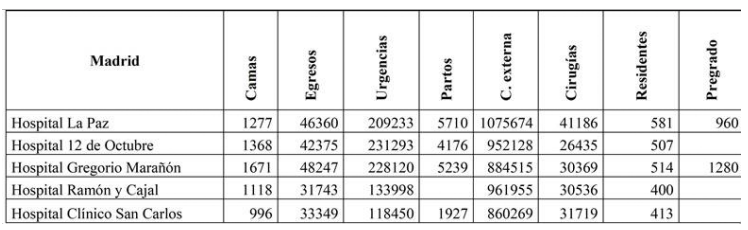

En la tabla 7 se calculan los indicadores de productividad de alumnos de pregrado, residentes y fellows en las universidades de Duke y de Arkansas.
Tabla 7.Indicadores de productividad calculados para Duke University Medical Center y AHC Arkansas (datos de 2015)

\begin{tabular}{|l|c|c|}
\hline \multicolumn{1}{|c|}{ Centro medico académico } & $\begin{array}{l}\text { Duke University } \\
\text { Medical Center }\end{array}$ & U. Arkansas \\
\hline Relación de alumnos de pregrado: docente & $\mathbf{0 , 2 2}$ & $\mathbf{0 , 4 9}$ \\
\hline $\begin{array}{l}\text { Relación de alumnos de postgrados residentes }+ \\
\text { fellows: docente }\end{array}$ & $\mathbf{0 , 5 2}$ & $\mathbf{0 , 5 5}$ \\
\hline Relación de alumnos de pregrado: cama & 0,45 & 1,54 \\
\hline Relación de residentes + fellows por cama & 1,05 & 1,75 \\
\hline Relación de funcionarios por cama & 7,98 & N. A \\
\hline Relación de egresos por cama al año & 42 & 58 \\
\hline Relación de egresos por alumnos de pregrado & 93 & 37 \\
\hline Relación de egresos por cada residente + fellows & 40 & 33 \\
\hline Relación de consultas por alumno de pregrado & 2474 & 626 \\
\hline Relación de consultas por residente + fellows & 1057 & 550 \\
\hline $\begin{array}{l}\text { Relación de consultas de urgencias por alumno de } \\
\text { pregrado }\end{array}$ & 164 & 83 \\
\hline $\begin{array}{l}\text { Relación de consultas de urgencias por residente + } \\
\text { fellows }\end{array}$ & 70 & 73 \\
\hline Relación de cirugias por alumno de pregrado & 93 & 23 \\
\hline Relación de cirugias por residentes + fellows & 39 & 20 \\
\hline Relación de laboratorios por cama & 5915 & N. A. \\
\hline
\end{tabular}

NotaN. A.: no aplica.

La revisión anterior muestra que la mayoría de los países tienen hospitales que ofrecen a sus universidades una proporción alta de camas y docentes por alumno, superiores a la relación 1:1. También muestra que la integración parece favorecer el modelo académico, que los hospitales son estatales, que la educación también es controlada por el gobierno y que en Estados Unidos, donde la mayor parte de universidades e instituciones que prestan servicios de salud son entidades privadas sin ánimo de lucro, existe una íntima relación entre universidades y hospitales. Creemos que, en el caso colombiano, el CMA es un modelo eficaz para mejorar la formación de los profesionales de la salud. Por lo tanto, hacemos una evaluación del Hospital Universitario San Ignacio y apuntamos a la integración de las misiones, respetando el marco legal vigente y las normas que rigen el concepto de hospital universitario en Colombia.

Al analizar la situación actual del CMA Hospital Universitario San Ignacio, teniendo en cuenta el número de alumnos de pre- y posgrado de los programas relacionados con la salud, el porcentaje de estudiantes que rotan en el hospital y las variables de número de camas, consultas externas, procedimientos quirúrgicos y consultas de urgencias, examinaremos hasta qué punto estos componentes y su relación se ajustan a las proporciones adecuadas para la formación del talento humano en el área de la salud. 


\section{Pregrado}

Los alumnos de pregrado asisten al CMA-HUSI en 3 momentos de su formación:

Semestres I a V: se trata de alumnos a los que deseamos exponer en una etapa temprana de su formación al ambiente clínico, en el que se construye y se fortalece el trabajo interdisciplinario con el personal de enfermería y se establecen rotaciones breves, cuya duración es de entre dos y tres días para estudiantes de primer semestre y puede ser de hasta dos semanas para estudiantes de cuarto semestre. Esta presencia de los alumnos en el hospital es muy importante para su formación; pero tiene un bajo impacto en volumen en la institución. Los alumnos de sexto semestre rotan extramuralmente en el componente de cuidado primario Javesalud.

Semestres VII-X: este es un componente muy importante del programa. El $63 \%$ de estas rotaciones se realizan en el hospital.

Internado: se realiza en 2 fases. En el semestre $\mathrm{XI}$ tiene lugar un internado extramural en el que solo el $3 \%$ de los alumnos rotan. En el semestre XII tiene lugar un internado universitario que se realiza en el hospital y en el que participa el $94 \%$ de los alumnos (tabla 8).
Tabla 8.Análisis de los indicadores de producción del Hospital Universitario San Ignacio respecto a alumnos de pregrado, residentes y fellows, 2016

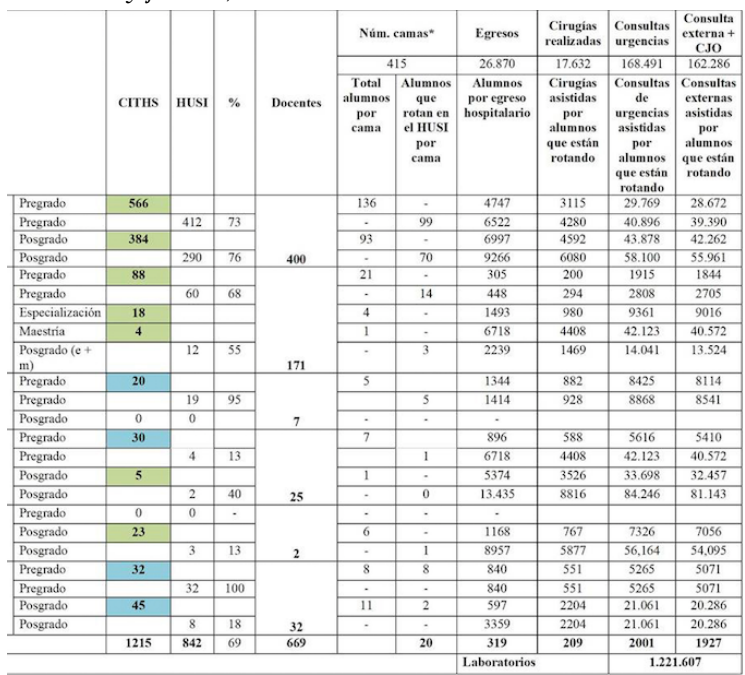

Notas *Promedio diario de camas disponibles (incluye camillas urgencias). CITHS: Comisión Intersectorial Talento Humano en Salud. HUSI: Hospital Universitario San Ignacio.

Javesalud es una institución de primer nivel vinculada a la Pontificia Universidad Javeriana. Es, asimismo, un CMA de primer nivel. En la institución rotan simultáneamente 145 estudiantes, tanto de pregrado como de posgrado. En cuanto a la producción de actividades en el 2015, las cifras se registran en la tabla 9.

Tabla 9.Cifras de Javesalud

\begin{tabular}{|l|r|}
\hline \multicolumn{1}{|c|}{ Grupo } & \multicolumn{1}{c|}{ Cantidad } \\
\hline Ayudas terapéuticas & 52.029 \\
\hline Consulta externa, medicina general & 103.851 \\
\hline Consulta externa, medicina especializada & 105.550 \\
\hline Consulta externa, odontología & 19.341 \\
\hline
\end{tabular}

La tabla 10 muestra cómo quedan los indicadores de la Facultad de Medicina de la Pontificia Universidad Javeriana, una vez que se agregan estas cifras.

Tabla 10.Relación comparada de indicadores de CMA HUSI + Javesalud

\begin{tabular}{|l|c|c|c|}
\hline & $\begin{array}{c}\text { Cuidado } \\
\text { primario }\end{array}$ & $\begin{array}{c}\text { Alta } \\
\text { complejidad }\end{array}$ & \multirow{2}{*}{ CMA } \\
\cline { 1 - 2 } CMavesalud & HUSI & \\
\hline Profesores clínicos de medicina & 40 & 431 & 471 \\
\hline Otros estudiantes de ciencias de la salud & 185 & 185 & 185 \\
\hline Camas & 0 & 400 & 400 \\
\hline Promedio de pacientes hospitalizados & 0 & 450 & 450 \\
\hline Egresos & 0 & 25.017 & 25.017 \\
\hline Consulta externa & 209.401 & 235.428 & 444.829 \\
\hline Consultas de urgencias & 0 & 176.547 & 176.547 \\
\hline Cirugías & 0 & 16.759 & 16.759 \\
\hline Laboratorios clínicos & 52.029 & 1.156 .992 & 1.209 .021 \\
\hline Partos & 0 & 1607 & 1607 \\
\hline
\end{tabular}


A partir lo expuesto en la tabla 8 sobre la distribución de alumnos respecto a las actividades asistenciales, presentamos los siguientes tres escenarios para someterlos a análisis:

1. Todos los alumnos de pregrado de la Facultad de Medicina y los residentes rotan en el CMA (HUSI + Javesalud).

2. Todos los alumnos de pregrado de la Facultad de Medicina y los residentes rotan en el CMA (HUSI + Javesalud), excepto los alumnos de primero a cuarto semestres, que no desarrollan actividades hospitalarias, y los internos del semestre XI.

3. Solo los alumnos de pregrado de la Facultad de Medicina y los residentes rotan en el CMA (HUSI + Javesalud).

Para cada uno de los escenarios propuestos, la tabla 11 muestra los indicadores:

Tabla 11.CMA Hospital Universitario San Ignacio + Javesalud, 2015

\begin{tabular}{|l|c|c|c}
\hline \multicolumn{1}{|c|}{ Centro médico académico } & $\mathbf{1}$ & $\mathbf{2}$ & $\mathbf{3}$ \\
\hline Relación de alumnos de pregrado: docente & $\mathbf{2 , 2 6}$ & $\mathbf{1 , 3 9}$ & $\mathbf{1 , 1 1}$ \\
\hline $\begin{array}{l}\text { Relación de alumnos de postgrados residentes + } \\
\text { fellows: docente }\end{array}$ & $\mathbf{0 , 6 4}$ & $\mathbf{0 , 6 4}$ & $\mathbf{0 , 6 4}$ \\
\hline Relación de alumnos de pregrado: cama & 2,66 & 1,64 & 1,30 \\
\hline Relación de residentes + fellows por cama & 0,75 & 0,75 & 0,75 \\
\hline Relación de egresos por cama al año & 62 & 62 & 62 \\
\hline Relación de egresos por alumnos de pregrado & 23 & 38 & 48 \\
\hline Relación de egresos por cada residente + fellow & 83 & 83 & 83 \\
\hline Relación de consultas por alumno de pregrado & 417 & 679 & 853 \\
\hline Relación de consultas por residente + fellows & 1482 & 1482 & 1482 \\
\hline $\begin{array}{l}\text { Relación consultas de urgencias por alumno de } \\
\text { pregrado }\end{array}$ & 165 & 269 & 338 \\
\hline $\begin{array}{l}\text { Relación de consultas de urgencias por residente + } \\
\text { fellow }\end{array}$ & 588 & 588 & 588 \\
\hline Relación de cirugías por alumno de pregrado & 15 & 25 & 32 \\
\hline Relación de cirugias por residentes + fellows & 55 & 55 & 55 \\
\hline Relación de laboratorios por cama & 3022 & 3022 & 3022 \\
\hline
\end{tabular}

Para desarrollar plenamente el CMA Hospital Universitario San Ignacio se requiere una institución de segundo nivel de, al menos, 140 camas y una producción proporcional a la que tiene esta institución en la actualidad y que se ha presentado en estas páginas. Así, los sitios de práctica formativa se volverían autosuficientes y podrían cubrir las necesidades de la Facultad de Medicina (tablas 12 y 13 ).
Tabla 12.Desarrollo del centro médico académico del Hospital Universitario San Ignacio

\begin{tabular}{|l|c|c|c|c|}
\hline \multirow{2}{*}{$\begin{array}{c}\text { CMA HUSI + Javesalud + segundo } \\
\text { Nivel }\end{array}$} & $\begin{array}{c}\text { Cuidado } \\
\text { primario }\end{array}$ & $\begin{array}{c}\text { Mediana } \\
\text { complejida } \\
\text { d }\end{array}$ & $\begin{array}{c}\text { Alta } \\
\text { complejidad }\end{array}$ & \multirow{2}{*}{ CMA } \\
\cline { 2 - 4 } Javesalud & $\mathbf{2}$ nivel & HUSI & \\
\hline Profesores clínicos de medicina & 40 & 151 & 431 & 622 \\
\hline $\begin{array}{l}\text { Otros estudiantes de ciencias de la } \\
\text { salud }\end{array}$ & 185 & 65 & 185 & 435 \\
\hline Camas & 0 & 140 & 400 & 540 \\
\hline Promedio de pacientes hospitalizados & 0 & 158 & 450 & 608 \\
\hline Egresos & 0 & 8778 & 25.017 & 33.795 \\
\hline Consulta externa & 209401 & 82606 & 235.428 & 527.435 \\
\hline Consultas de urgencias & 0 & 61946 & 176.547 & 238.493 \\
\hline Cirugías & 0 & 5880 & 16.759 & 22.639 \\
\hline Laboratorios clínicos & 52029 & 405962 & 1.156 .992 & 1.614 .983 \\
\hline Partos & 0 & 564 & 1.607 & 2.171 \\
\hline
\end{tabular}

Tabla 13.CMA Hospital Universitario San Ignacio + Javesalud + proyección segundo nivel

\begin{tabular}{|l|c|c|c|}
\hline \multicolumn{1}{|c|}{ Centro médico académico } & $\mathbf{1}$ & $\mathbf{2}$ & $\mathbf{3}$ \\
\hline $\begin{array}{l}\text { Relación de alumnos de pregrado: } \\
\text { docente }\end{array}$ & $\mathbf{1 , 7 1}$ & $\mathbf{1 , 0 5}$ & $\mathbf{0 , 8 4}$ \\
\hline $\begin{array}{l}\text { Relación de alumnos de posgrados, } \\
\text { residentes + fellows: docente }\end{array}$ & $\mathbf{0 , 4 8}$ & $\mathbf{0 , 4 8}$ & $\mathbf{0 , 4 8}$ \\
\hline $\begin{array}{l}\text { Relación de alumnos de pregrado: } \\
\text { cama }\end{array}$ & 1,97 & 1,21 & 0,96 \\
\hline $\begin{array}{l}\text { Relación de residentes + fellows por } \\
\text { cama }\end{array}$ & 0,56 & 0,56 & 0,56 \\
\hline Relación de egresos por cama al año & 62,54 & 62,54 & 62,54 \\
\hline $\begin{array}{l}\text { Relación de egresos por alumnos de } \\
\text { pregrado }\end{array}$ & 31,73 & 51,60 & 64,87 \\
\hline $\begin{array}{l}\text { Relación de egresos por cada } \\
\text { residente + fellow }\end{array}$ & 112,65 & 112,65 & 112,65 \\
\hline $\begin{array}{l}\text { Relación de consultas por alumno de } \\
\text { pregrado }\end{array}$ & 495,24 & 805,24 & 1012,35 \\
\hline $\begin{array}{l}\text { Relación de consultas por residente }+ \\
\text { fellows }\end{array}$ & 1758,12 & 1758,12 & 1758,12 \\
\hline $\begin{array}{l}\text { Relación de consultas de urgencias } \\
\text { por alumno de pregrado }\end{array}$ & 223,94 & 364,11 & 457,76 \\
\hline $\begin{array}{l}\text { Relación de consultas de urgencias } \\
\text { por residente + fellow }\end{array}$ & 794,98 & 794,98 & 794,98 \\
\hline $\begin{array}{l}\text { Relación de cirugias por alumno de } \\
\text { pregrado }\end{array}$ & 21,26 & 34,56 & 43,45 \\
\hline $\begin{array}{l}\text { Relación de cirugias por residentes + } \\
\text { fellows }\end{array}$ & 75,46 & 75,46 & 75,46 \\
\hline Relación de laboratorios por cama & 2988,77 & 4037,46 & 2988,77 \\
\hline
\end{tabular}

\section{Conclusiones}

Para enriquecer la discusión, exponemos los siguientes planteamientos, tomando como base la experiencia del Hospital Universitario San Ignacio:

La enseñanza de la medicina es distinta en cada país. De acuerdo con los objetivos de sus políticas públicas de salud y educación, se pueden encontrar diversos requisitos o recomendaciones para dimensionar los escenarios de la práctica clínica.

Lo que está fuera de toda duda es que no se puede hablar de calidad en la formación de talento humano en el campo de la salud teniendo en mente solo modelos teóricos y de simulación. Es indispensable disponer de IPS que 
permitan el entrenamiento práctico de los futuros profesionales.

En el mundo existe la tendencia a contar con hospitales universitarios acreditados y, en especial, a conformar CMA, para alcanzar una alta calidad en los procesos de educación médica. Esta es una estrategia para consolidar procesos docentes, asistenciales, investigativos y de extensión que brinden una mejor formación al talento humano en el área de la salud y que reporten un mayor beneficio a las sociedades del entorno.

Es bien sabido que el país sufre un déficit de profesionales y que debe mejorar la calidad de la educación. El estudio Los recursos humanos de la salud en Colombia. Balance, competencias y prospectiva, realizado para el Ministerio de la Protección Social por el Centro de Proyectos para el Desarrollo de la Pontificia Universidad Javeriana (Cendex) [16], señala que el déficit de médicos proyectado para 2011 era el siguiente: médicos generales, de 14.424 a 25.780; médicos internistas, de 206 a 480; cirujanos generales, de 217 a 422; pediatras, de 209 a 497; anestesiólogos, de 485 a 732; ortopedistas y traumatólogos, de 77 a 184; segunda especialidad, cirugía general, de 217 a 422; segunda especialidad, medicina interna, de 206 a 480, y segunda especialidad, pediatría, de 206 a 480.

Se requiere un gran esfuerzo para consolidar redes de prestadores de servicios de salud con vocación y capacidad docente que resuelvan este déficit y brinden una formación de buena calidad. Estas redes estarían conformadas por CMA, hospitales universitarios y centros de práctica que tengan una producción y unas capacidades mínimas para que los alumnos de pre-y posgrado realicen sus prácticas de forma adecuada.

Con la promulgación de la Ley Estatutaria de Salud (Ley 1751 de 2015) y la próxima entrada en vigor del nuevo Modelo de Atención Integral en Salud (MIAS), expedido en el 2016 y, por el momento, en proceso de reglamentación e implementación, las entidades educativas y asistenciales deben ajustar los modelos de docencia y servicio. Gracias a estos ajustes, debe quedar claro cuáles son los escenarios de nivel primario y complementario, y, sobre todo, debe hacerse hincapié en la preparación de los niveles primarios y ambulatorios, aplicando el enfoque de medicina familiar para educar a los estudiantes de pregrado. El objetivo es formar a los profesionales que el país requiere a corto y mediano plazo.

Como propuesta para la discusión, la tabla 14 plantea los indicadores de capacidad mínima, a partir de la fluctuación de valores entre los centros médicos de las universidades de Duke y de Arkansas, y del agregado HUSI-Javesalud:

Tabla 14.Centro médico académico colombiano

\begin{tabular}{|l|r|}
\hline Relación de alumnos de pregrado: docente & $1-2: 1$ \\
\hline Relación de alumnos de posgrados residentes + fellows: docente & $1: 1$ \\
\hline Relación de alumnos de pregrado: cama & $1-2: 1$ \\
\hline Relación de residentes + fellows por cama & $1: 1$ \\
\hline Relación de funcionarios por cama & $7: 1$ \\
\hline Relación de egresos por alumnos de pregrado & $40-50: 1$ \\
\hline Relación de egresos por cada residente + fellow & $70: 1$ \\
\hline Relación de consultas por alumno de pregrado & $400: 1$ \\
\hline Relación de consultas por residente + fellows & $400: 1$ \\
\hline Relación de consultas de urgencias por alumno de pregrado & $250: 1$ \\
\hline Relación de consultas de urgencias por residente + fellow & $400: 1$ \\
\hline Relación de cirugias por alumno de pregrado & $25-30: 1$ \\
\hline Relación de cirugias por residentes + fellows & $50: 1$ \\
\hline Relación de laboratorios por cama & $2500: 1$ \\
\hline
\end{tabular}

\section{Referencias}

1. Maastricht University. Faculty
of Health, Medicine and Life Sciences [internet]. Disponible en: https://www.maastrichtuniversity.nl/ab out-um/faculties/faculty-health-medicine-a nd-life-sciences

2. National University of Singapore. How can we help you today [internet]. Disponible en: h ttp://www.nus.edu.sg/uhc/

3. Top Universities. QS World University Rankings by Subject 2016-Medicine [internet]. Disponible en: http://www.topu niversities.com/university-rankings/univers ity-subject-rankings/2016/medicine

4. Bombí JA. Teaching in Spanish medical schools. Med Teach. 2003 Jul;25(4):428-32.

5. Nepal Medical Council. Accreditation Standards for the MBBS (Bachelor of Medicine and Bachelor of Surgery): Degree Program for Institutions Admitting 100 students Annually [internet]. Disponible 
en: http://www.nmc.org.np/downloads/932 94.pdf

6. Medical Council of India. Minimum Standard Requirements for the Medical College for 150 Admissions Annually Regulations, 1999 (Part III, Section 4). Gazette of India. 1999, 29th April. New Delhi.

7. Montenegro-Idrogo J, Montañez-Valverde R, Sánchez-Tonohuye J. Sobredemanda del campo clínico para estudiantes de medicina. Rev Peru Med Exp Salud Pública. 2012;29(1):149-67.

8. Gobierno de Chile. Resolución 254 aprueba Norma General Técnica y Administrativa que regula la relación asistencial-docente y establece criterios para la asignación y uso de los campos para la formación profesional y técnica en el Sistema Nacional de Servicios de Salud y deroga Resolución Exenta n. 9418 del 10.03.10 [internet]. 9 de julio de 2012. Disponible en: http://ssmaule.redsalud.gob.cl/index.ph p/unidad-docente-asistencial/send/38-unid ad-docente-asistencial/760-resolucion-254 -aprueba-ngta-relacion-asistencial-docente $-09-07-12$

9. Ministerio de Salud de Chile. Capacidad formadora [internet]. 7 de octubre de 2015. Disponible en: http://web.minsal.cl/capacid ad-formadora/

10. Norma Oficial Mexicana NOM-234SSA1-2003. Utilización de campos clínicos para ciclos clínicos e internado de pregrado.

11. Ministerio de Salud Pública (MSP), Consejo de Educación Superior (CES). Norma técnica para unidades asistenciales-docentes. Quito: Ministerio de Salud Pública, Dirección Nacional de Normatización, Dirección Nacional de Normatización del Talento Humano; 2013.

12. Universidad de Antioquia. IPS Universitaria: servicios de salud [internet]. Disponible en: http://portal.ipsuniversitaria.com.co/D ocencia/docs/informe2009_05_2010.pdf

13. DukeHealth. [internet]. Disponible en: http://corporate.dukemedicine.org/Abo utUs/Facts_and_Statistics/
14. University of Arkansas for Medical Sciences. Fast facts [internet]. Disponible en: http:// web.uams.edu/about/fast-facts/

15. Comunidad de Madrid, Observatorio de Resultados. Informe de Hospitales, años 2011-2013. Madrid: Comunidad de Madrid, Consejería de Sanidad, Servicio Madrileño de Salud; 2014.

16. Ruiz F, Matallana MA, Amaya JL, Vásquez ME, Parada LA, Piña MR. Los recursos humanos de la salud en Colombia: balance, competencias y prospectiva. Bogotá: Ministerio de la Protección Social de Colombia; 2008. 